\title{
Diferenciación morfológica y por ISSR (Inter simple sequence repeats) de especies del género Plukenetia (Euphorbiaceae) de la Amazonía peruana: propuesta de una nueva especie
}

\author{
Differentiation morphological and by Inter simple sequence repeats \\ (ISSR) of species of genus Plukenetia (Euphorbiaceae) from Peruvian \\ Amazon: suggestion for a new species
}

\section{Ángel Rodríguez ${ }^{1 *}$, Mike Corazon-Guivin ${ }^{1}$, Danter Cachique ${ }^{1}$, Kember Mejía ${ }^{1}$, Dennis Del Castillo ${ }^{1}$, Jean-François Renno ${ }^{2}$, Carmen García-Dávila ${ }^{1}$}

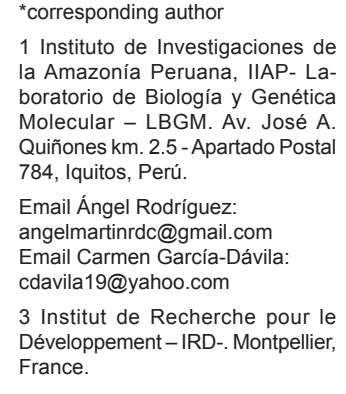
France.

\section{Resumen}

En el presente trabajo se estudian cinco especies del género Plukenetia de la Amazonía peruana: $P$. brachybotrya, P. loretensis, P. polyadenia, P. huayllabambana, P. volubilis (procedencia San Martín); y de un supuesto morfotipo ( $P$. volubilis, procedencia Cusco). Los 126 especímenes estudiados fueron identificados mediante claves de caracteres morfológicos (formas de hojas, tallos y semilla; posición de glándulas basilaminares) y posteriormente mediante marcadores moleculares ISSR (CAA, CAG, GACA). Los análisis morfológicos permitieron separar las cinco especies descritas: P. brachybotrya, P. loretensis, P. polyadenia, P. volubilis y $P$. huayllabambana. Los dos supuestos morfotipos de $P$. volubilis fueron discriminados por la posición de las glándulas, tamaño de semillas y forma del tallo. Los resultados proporcionados por el Análisis Factorial de Correspondencia (AFC) y corroborados por el Índice de fijación $\left(\mathrm{F}_{\mathrm{ST}}\right)$, distancia genética y el dendograma estimado por el método UPGMA, evidencian una fuerte diferenciación entre los seis taxa, corroborando la identidad taxonómica molecular de las cinco especies ya descritas morfológicamente. Además, los resultados (Fst y la distancia genética) indicarian que $P$. volubilis (del Cusco) podría ser una nueva especie de Sacha Inchi, aún no descrita para la ciencia.

Palabras clave: Sacha inchi, ISSR, Plukenetia, Biodiversidad, Amazonía.

\section{Abstract}

In this work, five taxa of the genus Plukenetia from Peruvian Amazon: P. brachybotrya, P. loretensis, $P$. polyadenia, P. huayllabambana, $P$. volubilis (from San Martin) and one morphotype ( $P$. volubilis, from Cusco) were studied. The 126 specimens used were identified by morphological keys (shapes of leaves, stems and seed; basilaminar glands position) and by ISSR molecular markers (CAA, CAG, GACA). The morphological analysis of the different taxa studied allowed us to clearly identify five described species: P. brachybotrya, P. loretensis, P. polyadenia, P. volubilis (from San Martin) and Plukenetia huayllabambana. Both morphotypes of $P$. volubilis (San Martin and Cusco) were distinguished for the position of the glands, seed size and shape of the stem. The results provided by Correspondence Factorial Analysis (AFC) and supported by the fixation index (Fst), genetic distance and the dendogram estimated by the UPGMA method, show a strong differentiation among the six taxa, corroborating the molecular taxonomic identity of the five species morphologically described. Also, results (Fst and Genetic distance) suggest that $P$. volubilis (from Cusco) could be a new Sacha Inchi species, not yet described to science.

Keywords: Sacha inchi, ISSR, Plukenetia, Biodiversity, Amazon.

\section{Introducción}

La diversidad de pisos ecológicos presentes en la Amazonía peruana, ha permitido a través de los siglos, la domesticación de numerosas especies de plantas nativas con una alta variabilidad genética (Zapata 2003). La impresionante diversidad biológica hace que muchas de estas especies no hayan sido estudiadas en profundidad, desconociendo aún muchas características biológicas, genéticas y fitoquímicas. Plukenetia volubilis L., sacha inchi es una planta nativa de la Amazonía peruana, cuyas semillas presentan altos contenidos de proteínas, ácidos grasos (esenciales: omegas 3, 6; omega 9) y vitamina E (tocoferoles y tocotrienoles) en cantidades significativamente mayores que otras semillas de oleaginosas como el maní, palma, soya, maíz, colza y girasol (Hamaker 1992).

El valor del sacha inchi radica no sólo en los aspectos alimenticios, culturales e históricos, sino también en su rentabilidad económica. Siendo un cultivo con potencial rendimiento económico y grandes posibilidades de industrialización (Arévalo
1995), viene siendo intensamente cultivado; sin embargo, se observa una alta variabilidad genética, morfológica y fitoquímica, lo que ha generado que en el proceso de expansión del cultivo se hayan llegado a confundir especies del género Plukenetia. Hasta el año 2008 fueron descritas para la Amazonía peruana, cuatro especies en base a caracteres morfológicos: P. volubilis L., P. brachybotrya Müll. Arg., P. loretensis Ule, y P. polyadenia Müll. Arg. Recientemente fue descrita, para la región Amazonas, P. huayllabambana Bussmann, C. Téllez \& A. Glenn. Nuestras observaciones en la morfología de algunas colecciones biológicas del Herbarium Amazonense (AMAZ) indican cierta sobreposición en algunos de los caracteres de estas especies, lo que podría dificultar su correcta determinación.

Los marcadores moleculares constituyen poderosas herramientas para esclarecer cuestiones no resueltas mediante la taxonomía morfológica y para determinar los límites taxonómicos. Así, el ADN han sido utilizado para comprobar la sistemática morfológica o para cuestionarla (Judd et al. 1999). La litera- 
tura registra la utilización de marcadores ISSR en estudios de validación de la información proporcionada por la taxonomía morfológica. Por ejemplo, Chennaoui-Kourda et al. (2007) utilizando marcadores ISSR en el análisis de la relación entre Sulla spinosissima y $S$. capitata, mostraron la existencia de una gran diferenciación genética entre estas dos especies; mientras que Shi et al. (2006) en el análisis molecular de 20 especies de Lycoris, reconocieron cuatro grandes grupos de especies, lo que resultó congruente con sus observaciones morfológicas y citológicas. Resultados similares acerca de marcadores ISSR fueron encontrados en trigo, cebada, algodón y otras plantas (Chen et al. 2007, Yockteng et al. 2003, Xu \& Sun 2001, Raina et al. 2001, Hang et al. 2003, Liu 1999). Así mismo Angiosperm Phylogeny Group (APG 2003), viene apoyando desde la década de los noventa el uso de marcadores moleculares en estudios filogenéticos de plantas, principalmente con la finalidad de proporcionar una nueva visión filogenética en la sistemática de plantas con flores.

En este sentido, el presente estudio tuvo como objetivo contribuir a la delimitación taxonómica de seis taxa del género Plukenetia identificadas con claves morfológicas y mediante análisis de su polimorfismo genético, empleando la técnica molecular ISSR. La información producida en el presente trabajo servirá de base a planes de manejo y mejoramiento genético.

\section{Material y métodos}

\section{Muestras biologicas}

Se colectaron muestras biológicas de 126 individuos pertenecientes a cinco especies: Plukenetia huallabambana (Chirimoto, Región Amazonas; 18M 0229606, UTM 9279162), P. polyadenia (Pevas, Región Loreto; 19M 0174043, UTM 9631195), $P$. loretensis y P. brachybotrya (Puerto Almendras, Región Loreto; 18M 0680833, UTM 9576661), Plukenetia volubilis (Shica, Región San Martín; 18M 0315372, UTM 9301077) y un último morfotipo conocido por los agricultores también como Plukenetia volubilis y que proviene de la localidad de Echarati en la Región de Cusco (18M 0765342, UTM 8583639).

Las muestras botánicas colectadas (hojas, flores y frutos) de cada taxón, fueron utilizadas para el montaje de exsicatas y la determinación mediante claves taxonómicas. Las muestras botánicas fueron depositadas como referencia de este estudio en el Herbarium Amazonense (AMAZ), de la Universidad Nacional de la Amazonía peruana - UNAP.

\section{Extracción y amplificación de ADN}

La extracción fue realizada a partir de tejido foliar (conservados con Sulfato de Calcio Anhidro), mediante el método de extracción CTAB (Doyle \& Doyle 1987). La amplificación del ADN se realizó con los primers CAA (CAACAACAACAACAA), CAG (CAGCAGCAGCAGCAG) y GACA (GACAGACAGACAGACA), diseńados por Bornet y Branchard (2001); de acuerdo a la técnica Inter Simple Sequence Repeat-ISSR (Zietkiewicz et al. 1994). Las amplificaciones fueron realizadas en volúmenes finales de $25 \mu \mathrm{L}$ conteniendo: $100 \mathrm{ng} / \mu \mathrm{L}$ de ADN molde, $5 \mathrm{U} / \mu \mathrm{L}$ de Taq polimerasa, $5 \mathrm{X}$ Buffer, $25 \mathrm{mM} \mathrm{MgCl}_{2}$, $10 \mathrm{mM}$ dNTPs, $10 \mu \mathrm{M}$ de primer y agua ultrapura. Las condiciones de temperatura fueron: una desnaturalización inicial a $95^{\circ} \mathrm{C}$ x 1 min., seguida de 27 ciclos consistentes en: desnaturalización $\left(94^{\circ} \mathrm{C} \times 1 \mathrm{~min}\right.$.), hibridación $\left(37-60^{\circ} \mathrm{C}\right.$, adecuada a cada primer $\mathrm{x} 1 \mathrm{~min}$.), elongación ( $72^{\circ} \mathrm{C} \mathrm{x} 4 \mathrm{~min}$.), seguido de una extensión final a $72{ }^{\circ} \mathrm{C}$ x $7 \mathrm{~min}$. La verificación preliminar de la amplificación fue realizada en geles de agarosa $2 \%$ teñidos con bromuro de etidio $(10 \mathrm{mg} / \mathrm{mL})$. Posteriormente se verificó el polimorfismo (diferenciación) entre las muestras en geles de poliacrilamida al $6 \%$, teñidos mediante tinción argéntica de acuerdo al método Rabat.

\section{Interpretación y tratamiento de datos}

Se realizó una matriz binaria en base a la presencia (1) y ausencia (0) de las bandas observadas (polimórficas) entre los individuos. La diversidad genética entre los diferentes taxa (especies morfológicas y morfotipo) de Plukenetia fue visualizada mediante el Análisis Factorial de Correspondencia (AFC), la diferenciación entre ellas fue estimada en base al índice de fijación $\left(\mathrm{F}_{\mathrm{ST}}\right)$ propuesto por Weir y Cockerham (1984). Estos análisis fueron realizados con la ayuda del software GENETIX versión 4.05 (Belkhir et al. 2004). Las relaciones entre las agrupaciones fueron establecidas en base a un dendrograma (método UPGMA), elaborado a partir de la distancia genética (D) obtenida según Reynolds et al. (1983), así como los valores de Bootstrap (calculados en base a 1000 repeticiones) fueron obtenidos con la ayuda de PHYLIP versión 3.5 (Felsenstein 1993). Los árboles fueron visualizados con ayuda del software TREVIEW (Page 1996).

\section{Resultados}

\section{Caracterización morfológica y tratamiento taxonómico}

En base a las claves taxonómicas de Gillespie (1993) se logró determinar cuatro especies morfológicas entre los taxa estudiados: P. loretensis, $P$. brachrybotrya, $P$. polyadenia, y $P$. volubilis (procedencia San Martín); y P. huayllabambana en base a las descripciones de Bussmann et al. (2009). Observándose qué P. volubilis (procedencia Cusco) presenta algunas diferencias morfológicas (hojas, fruto y semillas) con relación a las especies antes mencionadas (Tabla 1).

Las características morfológicas diferenciales más saltantes la encontramos en la forma de las semillas de los diferentes grupos de Plukenetia (Fig. 1), que van desde redondeadas hasta ligeramente lenticuladas; y diferencias en la testa de las semillas, de aspecto liso en cuatro de los seis taxa [P. loretensis, P. brachybotrya, P. volubilis (procedencia San Martín) y P. polyadenia], y rugosa en $P$. huayllabambana y $P$. volubilis (procedencia Cusco). Asimismo se observó una considerable variación en el tamaño de las semillas.

\section{Caracterización molecular}

El resultado del Análisis Factorial de Correspondencia (AFC) muestra los seis taxa estudiados claramente diferenciados a nivel genético sin sobreposición alguna entre ellos (Fig. 2: A, B, C). $\mathrm{El}$ análisis del $\mathrm{F}_{\mathrm{ST}}$ entre pares de taxa (Tabla 2) mostró un alto grado de diferenciación entre todos los taxa analizados (elevada significancia, $p=0)$. P. brachybotrya y $P$. volubilis (procedencia Cusco) tienen el $\mathrm{F}_{S T}$ más elevado $\left(\mathrm{F}_{S \mathrm{~T}}=0,98491 ; \mathrm{p}=0\right)$. Mientras que $P$. huayllabambana y $P$. volubilis (procedente de de San Martín) se mostraron como los taxa más cercanos $\left(\mathrm{F}_{\mathrm{ST}}=0,89562\right.$, $p=0)$. Los $\mathrm{F}_{\mathrm{ST}}$ por pares fueron estadísticamente significativos.

Las distancias genéticas (Tabla 2) estimadas según Reynolds et al. (1983) muestran diferencias marcadas entre cada par de agrupaciones. Siendo que $P$. volubilis (procedencia San Martín) y $P$. huayllabambana presentaron la menor distancia encontrada $(\mathrm{D}=2,25973)$. En cuanto que $P$. brachybotrya y $P$. volubilis 
Tabla 1. Principales diferencias morfológicas de los seis taxa del género Plukenetia estudiados en la Amazonía peruana.

\begin{tabular}{|c|c|c|c|c|c|c|}
\hline \multirow[b]{2}{*}{$\begin{array}{l}\text { Caracteres } \\
\text { observados }\end{array}$} & \multicolumn{6}{|c|}{ Agrupaciones Plukenetia estudiadas } \\
\hline & P. loretensis & P. brachybotrya & P. polyadenia & $\begin{array}{l}\text { P. volubilis } \\
\text { (procedencia } \\
\text { San Martín) }\end{array}$ & $\begin{array}{l}\text { P. volubilis } \\
\text { (procedencia } \\
\text { Cusco) }\end{array}$ & P. huayllabambana \\
\hline $\begin{array}{l}\text { Glándulas } \\
\text { foliares } \\
\text { basilaminares }\end{array}$ & $\begin{array}{c}\text { Glándulas } \\
\text { basilaminares } \\
\text { en uno o más } \\
\text { pares próximas al } \\
\text { pecíolo }\end{array}$ & $\begin{array}{l}\text { Glándulas } \\
\text { basilaminares } \\
\text { numerosas } \\
\text { próximas al } \\
\text { pecíolo. }\end{array}$ & $\begin{array}{l}\text { Un único } \\
\text { promontorio } \\
\text { glandular }\end{array}$ & $\begin{array}{c}\text { Par de glándulas } \\
\text { basilaminares } \\
\text { próximas al } \\
\text { pecíolo. }\end{array}$ & $\begin{array}{l}\text { Par de glándulas } \\
\text { basilaminares } \\
\text { relativamente } \\
\text { distantes del } \\
\text { pecíolo. }\end{array}$ & $\begin{array}{l}\text { Par de glándulas } \\
\text { basilaminares } \\
\text { relativamente } \\
\text { distantes del } \\
\text { pecíolo. }\end{array}$ \\
\hline $\begin{array}{l}\text { Borde y base } \\
\text { de la hoja }\end{array}$ & $\begin{array}{l}\text { Borde crenado y } \\
\text { base caudada. }\end{array}$ & $\begin{array}{l}\text { Borde liso y base } \\
\text { caudada. }\end{array}$ & $\begin{array}{l}\text { Borde liso } \\
\text { y base } \\
\text { acuminada. }\end{array}$ & $\begin{array}{l}\text { Borde crenado y } \\
\text { base caudada. }\end{array}$ & $\begin{array}{l}\text { Borde aserrado y } \\
\text { base caudada. }\end{array}$ & $\begin{array}{l}\text { Borde crenado y } \\
\text { base caudada. }\end{array}$ \\
\hline Base del tallo & Redondeado & Redondeado & Aplanado & Redondeado & Redondeado & Hexagonal \\
\hline $\begin{array}{l}\text { Fruto } \\
\text { (cápsula) }\end{array}$ & $\begin{array}{l}\text { Cada carpelo con } \\
\text { cornículo agudo }\end{array}$ & $\begin{array}{l}\text { Cada carpelo } \\
\text { con un tubérculo } \\
\text { redondeado }\end{array}$ & $\begin{array}{l}\text { Cuadrangular } \\
\text { con ángulos } \\
\text { quillados }\end{array}$ & $\begin{array}{l}\text { Cuadrangular } \\
\text { con ángulos } \\
\text { quillados }\end{array}$ & $\begin{array}{l}\text { Cuadrangular con } \\
\text { ángulos quillados }\end{array}$ & $\begin{array}{l}\text { Cuadrangular con } \\
\text { ángulos quillados }\end{array}$ \\
\hline $\begin{array}{l}\text { Tamaño de la } \\
\text { cápsula }\end{array}$ & $\begin{array}{l}\text { Diámetro aprox. } \\
1,15 \mathrm{~cm} .\end{array}$ & $\begin{array}{l}\text { Diámetro aprox. } \\
1,15 \mathrm{~cm} .\end{array}$ & $\begin{array}{l}\text { Diámetro aprox. } \\
\text { de 6-11 cm. }\end{array}$ & $\begin{array}{l}\text { Diámetro aprox. } \\
\text { de } 5 \text { a } 6 \mathrm{~cm} .\end{array}$ & $\begin{array}{c}\text { Diámetro aprox. } 5 \\
\text { a } 6 \mathrm{~cm} .\end{array}$ & $\begin{array}{c}\text { Diámetro aprox. } 6 \\
\text { a } 8 \mathrm{~cm} \text {. }\end{array}$ \\
\hline $\begin{array}{l}\text { Superficie de } \\
\text { la semilla }\end{array}$ & Lisa & Lisa & Lisa & Lisa & Rugosa & Rugosa \\
\hline $\begin{array}{l}\text { Forma de la } \\
\text { semilla }\end{array}$ & Redondeada & Redondeada & Redondeada & Aplanada & $\begin{array}{l}\text { Ligeramente } \\
\text { aplanada }\end{array}$ & $\begin{array}{l}\text { Ligeramente } \\
\text { aplanada }\end{array}$ \\
\hline $\begin{array}{l}\text { Tamaño } \\
\text { semilla }\end{array}$ & $\begin{array}{c}\text { Media }=0,51 \mathrm{x} \\
0,42 \mathrm{~cm}\end{array}$ & $\begin{array}{c}\text { Media }=0,41 \mathrm{x} \\
0,39 \mathrm{~cm}\end{array}$ & $\begin{array}{c}\text { Media }=2,89 x \\
2,6 \mathrm{~cm}\end{array}$ & $\begin{array}{c}\text { Media }=2,01 \mathrm{x} \\
0,85 \mathrm{~cm}\end{array}$ & $\begin{array}{c}\text { Media }=2,01 \times \\
1,36 \mathrm{~cm}\end{array}$ & $\begin{array}{c}\text { Media }=2,38 \times 1,66 \\
\mathrm{~cm}\end{array}$ \\
\hline
\end{tabular}

(C)

(D)

(E)

(F)

(B)

(A)

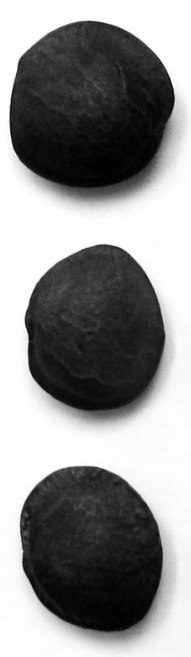

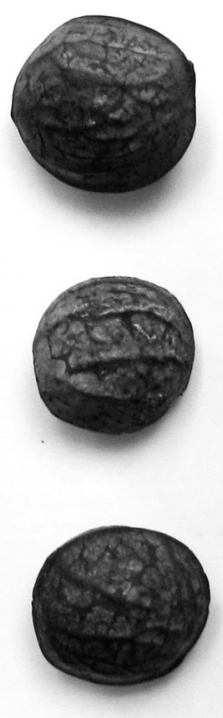
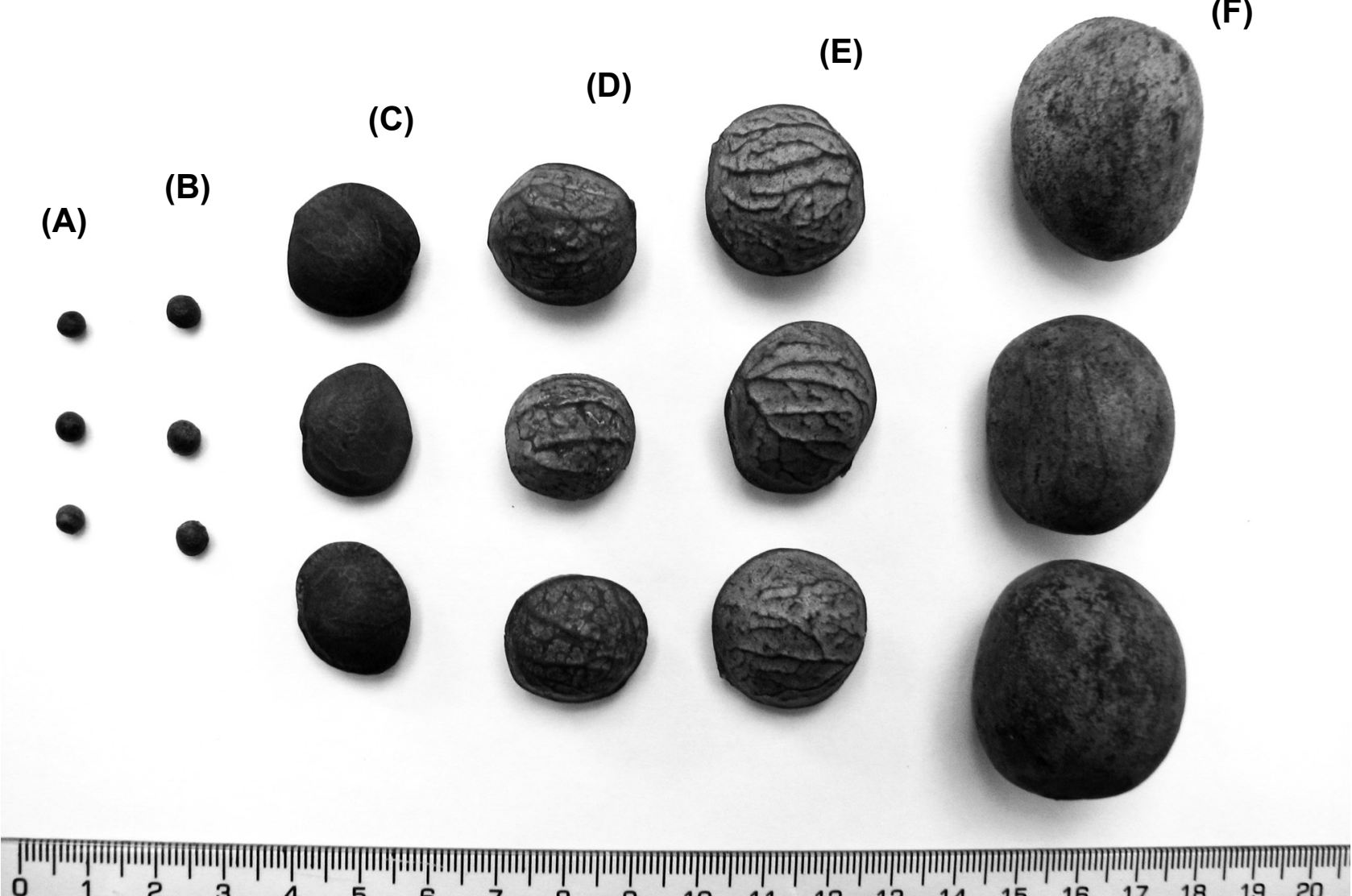

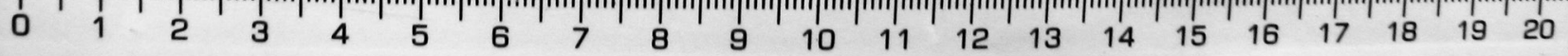

Figura 1. Semillas de de las seis agrupaciones del género Plukenetia estudiados en la Amazonía peruana: $\mathbf{A}=P$. brachybotrya; $\mathbf{B}=P$. loretensis; $\mathbf{C}=P$. volubilis (procedencia San Martín); $\mathbf{D}=P$. volubilis (procedencia Cusco); $\mathbf{E}=P$. huallaybambana; $\mathbf{F}=P$. polyadenia. 
(A)

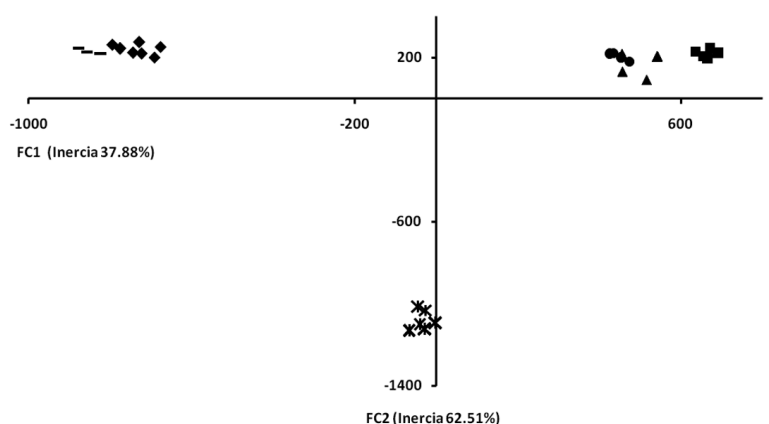

(B)

(C)

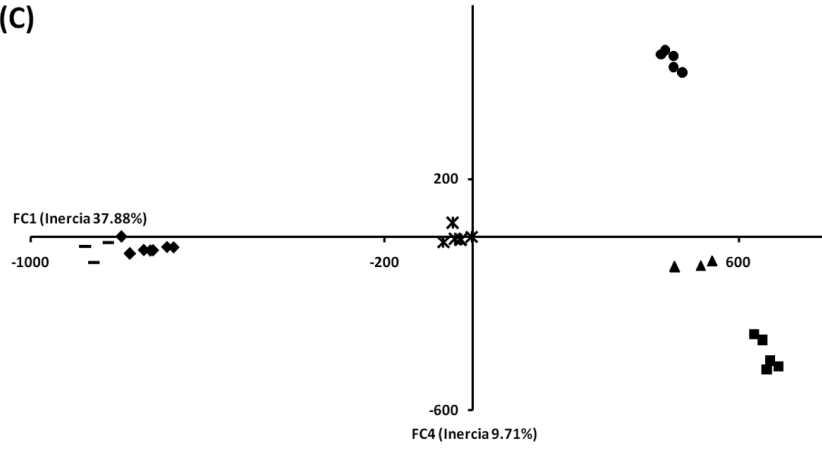

Figura 2. Proyección gráfica de los resultados del AFC: (A) ejes 1 y 2, (B) ejes 1 y $3,(C)$ ejes 1 y 4 para los individuos de de las seis agrupaciones de Plukenetia estudiados en la Amazonía peruana. $P$. huayllabambana $=\boldsymbol{\Lambda}, P$. polyadenia ${ }^{*}, P$. loretensis $=\star, P$. brachybotrya $=-, P$ volubilis (procedencia San Martín) $=\bullet, P$. volubilis (procedencia Cusco) $=\mathbf{m}$. Algunos puntos en los gráficos representan la sobreposición de más de un individuo. (procedencia Cusco) presentaron la mayor distancia genética $(\mathrm{D}=4,19378)$.

El dendograma obtenido mediante el método UPGMA (Fig. 3) muestra dos principales agrupaciones: la primera conformado por P. volubilis (procedencia San Martín), P. volubilis (procedencia Cusco) y $P$. huayllabambana (bootstrap $=100)$, dentro del cual se observa una sub-agrupación constituida por $P$. volubilis (procedencia San Martín) y P. huayllabambana (bootstrap=68); el segundo grupo lo conforman $P$. polyadenia, $P$. loretensis y $P$. brachybotrya (bootstrap $=100) ;$. brachybotrya y $P$. loretensis son las más cercanamente relacionadas (bootstrap=96).

\section{Discusión}

En la revisión de Plukenetia de Gillespie (1993) existen 11 especies neotropicales, las cuales pueden ser divididas en dos grupos informales de acuerdo a la forma del estilo, morfología del androceo, estructura de la exina del polen, tamaño del fruto y forma de la hoja. El primer grupo Cylindrophora, conformado por $P$. volubilis, $P$. lehmaniana, $P$. polyadenia y $P$. stipellata; y el segundo grupo, Euplukenetia con $P$. brachybotrya, $P$. loretensis y $P$. verrucosa. El género Plukenetia puede ser reconocido por la presencia de glándulas basilaminares en el lado adaxial de la lámina foliar, el fruto generalmente 4-lobado y el hábito frecuentemente trepador y algunas veces rastrero.

Un análisis primario de los datos morfológicos nos permitió ubicar los taxa de Plukenetia en estudio, en cinco especies: Plukenetia volubilis (procedencia San Martín), P. polyadenia, $P$. huyllabambana, P. loretensis y P. brachybotrya.

Plukenetia loretensis y P. brachybotrya fueron separadas de las demás con claridad por la presencia de un par a numerosas glándulas basilaminares cerca al pecíolo.

También se pudo observar un carácter diferencial en la base del tallo entre $P$. huayllabambana (forma hexagonal) y $P$. polyadenia (forma aplanada).

Las diferencias a nivel de fruto y semilla fueron las más notorias. El tamaño de la cápsula y las semillas, separan claramente los taxa: P. brachybotrya y P. loretensis tienen las menores dimensiones comparadas con los otros taxa, la superficie de sus semillas son lisas y el aspecto es redondeado para ambas especies;

Tabla 2. Estimación de Índices de Fijación $\left(F_{s t}\right)$ y Distancias genéticas (D) según Reynolds et al. (1983) para los seis taxa de Plukenetia estudiadas en la Amazonía peruana.

\begin{tabular}{|c|c|c|}
\hline Pares de taxa & $\mathbf{F}_{\mathrm{ST}}$ & $\mathbf{D}$ \\
\hline P. volubilis (procedencia San Martín) - P. volubilis (procedencia Cusco) & $0,92804^{* * *}$ & 2,63171 \\
\hline P. volubilis (procedencia San Martín) - P. huayllabambana & $0,89562 * * *$ & 2,25973 \\
\hline P. volubilis (procedencia San Martín) - P. polyadenia & $0,95347^{* * *}$ & 3,06761 \\
\hline P. volubilis (procedencia San Martín) - P. brachybotrya & $0,97088^{* * *}$ & 3,53633 \\
\hline P. volubilis (procedencia San Martín) - P. loretensis & $0,96092^{* * *}$ & 3,24215 \\
\hline P. volubilis (procedencia Cusco) - P. huayllabambana & $0,91025^{* * *}$ & 2,41075 \\
\hline P. volubilis (procedencia Cusco) - P. polyadenia & $0,96939 * * *$ & 3,48649 \\
\hline P. volubilis (procedencia Cusco) - P. brachybotrya & $0,98491^{* * *}$ & 4,19378 \\
\hline P. volubilis (procedencia Cusco) - P. loretensis & $0,97678 * * *$ & 3,76277 \\
\hline P. huayllabambana - P. polyadenia & $0,95738^{* * *}$ & 3,15540 \\
\hline P. huayllabambana - P. brachybotrya & $0,97519^{* * *}$ & 3,69661 \\
\hline P. huayllabambana - P. loretensis & $0,96564^{* * *}$ & 3,37093 \\
\hline P. polyadenia - P. brachybotrya & $0,97223^{* * *}$ & 3,58366 \\
\hline P. polyadenia - P. loretensis & $0,96298^{* * *}$ & 3,29635 \\
\hline P. brachybotrya - P. loretensis & $0,95991^{* * *}$ & 3,21657 \\
\hline
\end{tabular}




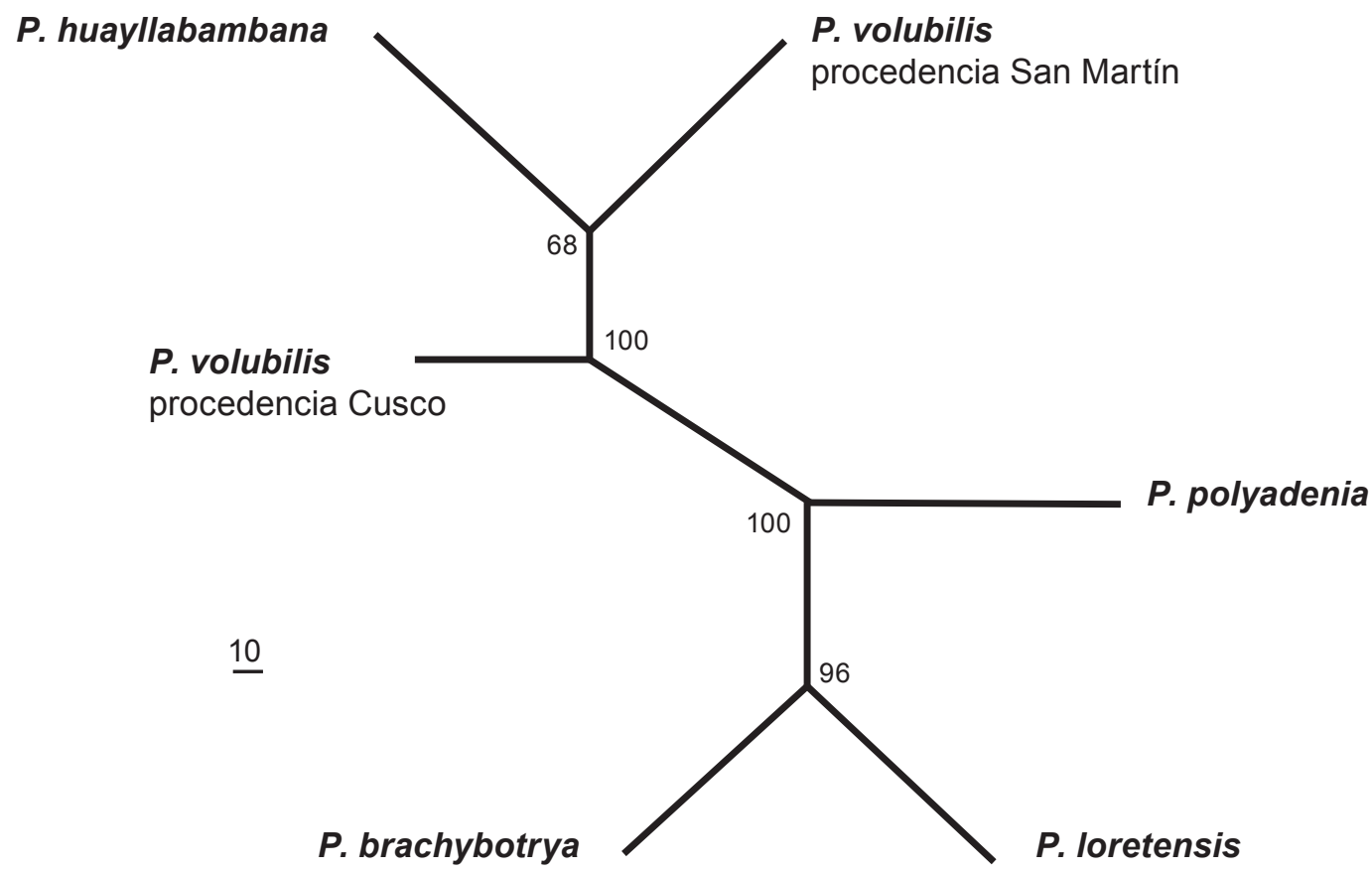

Figura 3. Dendograma de las seis agrupaciones del género Plukenetia estudiados en la Amazonía peruana. Elaborado mediante el método UPGMA a partir de los datos de distancia genética de Reynolds et al. (1983). Los números en las ramas representan los valores de bootstrap para 1000 repeticiones.

el carácter diagnóstico entre estas dos especies es la forma de la cápsula: en $P$. loretensis cada carpelo tiene un cornículo agudo, y en $P$. brachybotrya cada carpelo se muestra como un tubérculo redondeado.

Por otro lado, P. polyadenia es la especie que presentó el mayor tamaño de frutos (de 6 a $11 \mathrm{~cm}$ ) y semillas, estas semillas tienen forma redondeada, y la cápsula es de forma cuadrangular con ángulos quillados.

Las semillas de $P$. volubilis (procedencia San Martín) son de menor tamaño que las de $P$. huayllabambana y $P$. volubilis (procedencia Cusco), mientras que las de P. huayllabambana son considerablemente mayores que las de $P$. volubilis (procedencia San Martín y procedencia Cusco).

La variabilidad en los caracteres morfológicos en $P$. volubilis fueron observados por Manco (2006), quien reportó la existencia de 47 ecotipos y Arévalo (1995) que menciona ecotipos con diferencias considerables en cuanto al área de follaje, tamaño y forma de sus hojas y semillas. Estas variaciones observadas en los diferentes caracteres morfológicos imposibilitan muchas veces una determinación taxonómica segura, siendo necesaria la utilización de otras herramientas como por ejemplo los marcadores moleculares, para verificar si las diferencias observadas entre los taxa, representan variabilidad del carácter (plasticidad) o diferencias entre especies próximas.

Los resultados moleculares hicieron posible la distinción clara de seis taxa genéticos bien diferenciados y fuertemente estructurados (ilustrado con el análisis AFC). Confirmando la identidad genética de las cinco especies: $P$. brachybotrya, $P$. loretensis, P. polyadenia, P. volubilis (procedencia San Martín) y $P$. huayllabambana. El AFC además mostró que $P$. volubilis (procedencia San Martín), P. huayllabambana y P. volubilis (procedencia Cusco), son entidades genéticas diferentes entre sí, estos resultados fueron corroborados con los resultados de $\mathrm{F}_{\mathrm{ST}} \mathrm{y}$ distancia genética (Tabla 3) donde se observa que los valores encontrados entre estos tres grupos son similares a las encontradas entre las otras especies. Evidenciando la identidad genética de las seis agrupaciones estudiadas.

El dendograma obtenido a partir de las distancias genéticas para las especies amazónicas del género Plukenetia muestra la formación de dos agrupaciones principales: la primera conformada por P. volubilis (procedencia San Martín), P. huayllabambana y $P$. volubilis (procedencia Cusco), siendo que P. volubilis (procedencia San Martín) y P. huayllabambana se encuentran más cercanamente relacionados (distancia genética $=2,26$ ). La segunda agrupación engloba a $P$. polyadenia, $P$. loretensis y $P$. brachybotrya, siendo que estas dos últimas se encuentran más relacionadas en la topología (distancias genéticas: P. brachybotrya y $P$. loretensis $=3,22$ ).

Estas agrupaciones genéticas encontradas parecen presentar afinidad en cuanto la forma de la semilla, en el caso de la primera agrupación es relativamente aplanada, en cuanto que la segunda agrupación presenta semillas redondeada en las tres especies. Al contrario de la clasificación informal de las Plukenetia neotropicales (basado entre otros caracteres en el tamaño del fruto) mencionada por Gillespie (1993), donde P. polyadenia se encuentra junto a $P$. volubilis en el grupo Cylindrhophora.

Las relaciones obtenidas en este trabajo muestran que Plukenetia polyadenia se encuentra más cercana de $P$. brachybotrya y $P$. loretensis que son especies que presentan semillas pequeñas (media: $P$. loretensis $=0,51 \times 0,42 \mathrm{~cm} ;$ P. brachybotrya $=0,41 \mathrm{x}$ $0,39 \mathrm{~cm}$ ), demostrando que el tamaño de los frutos no puede ser considerado como carácter diagnostico (diferencial) para agrupar las especies estudiadas. 


\section{Conclusiones}

Los resultados moleculares obtenidos muestran un alto nivel de diferenciación entre los seis taxa de Plukenetia estudiados, corroborando la identidad taxonómica de las cinco especies de Plukenetia descritos hasta el momento para la Amazonía peruana: $P$. brachybotrya, $P$. loretensis, $P$. polyadenia $P$. volubilis y P. huayllabambana.

Así mismo, la divergencia genética encontrada entre las especies taxonómicamente descritas es igual a la encontrada entre los dos supuestos ecotipos de $P$. volubilis procedentes de San Martín y Cusco, sugiriendo que estas agrupaciones representan entidades genéticas separadas y no diferentes morfotipos dentro de $P$. volubilis, como se supuso al inicio de este estudio. Por lo tanto si consideramos las diferencias genéticas entre las especies ya descritas, y aceptamos el enlace entre nivel taxonómico y distancia genética, entonces este trabajo permitiría evidenciar una nueva especie de Sacha Inchi en la región de Cusco.

\section{Agradecimientos}

Al proyecto Innovación y Competitividad para el Agro Peruano - INCAGRO por el financiamiento otorgado para la realización de la presente investigación.

\section{Literatura citada}

APG, Angiosperm Phylogeny Group. 2003. An update of the Angiosperm Phylogeny Group classification for the orders and families of flowering plants: APG II. Botanical Journal of the Linnean Society 141: 399-436.

Arévalo G. 1995. El cultivo del sacha inchi (Plukenetia volubilis 1.) en la Amazonía. Programa Nacional de Investigación en Recursos Genéticos y Biotecnología - PRONARGEB, Estación Experimental El Porvenir - Tarapoto, Perú. 21 pp.

Belkhir K., P. Borsa, I. Chichi, et al. 2004. Genetix 4.05.2, logiciel sous windowns TM pour la genétique des populations. Laboratoire génome, populations, interactions, CNRS UMR 5000, Université de Montpellier II, Montpellier, France.

Bornet B. \& M. Branchard. 2001. Non anchored Inter Simple Sequence Repeat (ISSR) Markers: Reproducible and Specific Tools for Genome Fingerprinting; Plant Molecular Biology Reporter 19: 209-215.

Bussmann R.W., C. Téllez \& A. Glenn. 2009. Plukenetia huayllabambana sp. nov. (Euphorbiaceae) from the upper Amazon of Peru. Nordic Journal of Botany 27: 313-315.

Chen J., W.R. Gituru \& Q. Wang. 2007. A comparison of the extent of genetic variation in the endangered Sagittaria natans and its widespread congener S. trifolia. Aquatic Botany 87: 1-6.

Chennaoui-Kourda H., S. Marghali, M. Marrakchi \& N. Trifi-Farah. 2007. Genetic diversity of Sulla genus (Hedysarea) and related species using Inter-simple Sequence Repeat (ISSR) markers. Biochemical Systematics and Ecology 35: 682-688.

Doyle J.J. \& J.L. Doyle. 1987. A rapid DNA isolation procedure for small quantities of fresh leaf tissue. Phytochem. Bull. 19:11-15.
Felsenstein J. 1993. PHYLIP(Phylogeny Inference Package), version 3.5 c. Seattle: University of Washington.

Gillespie L.J. 1993. A synopsis of Neotropical Plukenetia (Euphorbiaceae) including two new species. Systematic Botany 18 (4): $575-592$.

Hamaker B.R., C. Valles, R. Gilman, et al. 1992. Amino Acid and Fatty Acid Profiles of the Inca Peanut (Plukenetia volubilis L.), Cereal Chem. 69: 461-463.

Hang A., C.S. Burton \& D.L. Hoffman. 2003. Use of intersimple sequence repeat (ISSR) polymorphisms to study genetic relationships in closely related North American malting barley cultivars. J. Genet. Breed. 91: 16-21.

Judd W., C. Campbell, E. Kellog, P. Stevens. 1999. Plant Systematics: A Phylogenetic Approach. Sinauer Associates, Inc. Sunderland, MA, USA. 464 pp.

Liu S. 1999. Cotton genetic mapping and QTL analysis using simple sequence repeat markers and the identification of their chromosome location. [MS Thesis]. New Mexico State University, Las Cruces, NM.

Manco E. 2006. Cultivo de sacha inchi. INIEA - SUDIRGEB - Estación Experimental Agraria "EL PORVENIR", Tarapoto, San Martín - Perú, 8 pp.

Page R.D.M. 1996. TreeView, Tree drawing software for Apple Macintosh and Microsoft Windows. Division of Environmental and Evolutionary Biology, Instituteof Biomedical and Life Sciences, University of Glasgow. Glasgow, Scotland, UK.

Raina S.N., V. Raini, T. Kojima, et al. 2001. RAPD and ISSR fingerprints as useful genetic markers for analysis of genetic diversity, varietal identification, and phylogenetic relationships in peanut (Arachis hypogaea) cultivars and wild species. Genome 44: 763-772.

Reynolds J.S., B.S. Weir \& C.C. Cockerham. 1983. Estimation of coancestry coefficient: basis for a short-term genetic distance. Genetics 105: 767-779.

Shi S, Y. Qiu, L. Wu \& C. Fu. 2006. Interspecific relationships of Lycoris (Amaryllidaceae) inferred from inter-simple sequence repeat data. Scientia Horticulturae 110: 285-291.

Weir B.S. \& C.C. Cockerham. 1984. Estimating F-statistics for the analysis of population structure. Evolution 38: 1358-1370.

Xu F. \& M. Sun. 2001. Comparative Analysis of Phylogenetic Relationships of Grain Amaranths and Their Wild Relatives (Amaranthus; Amaranthaceae) Using Internal Transcribed Spacer, Amplified Fragment Length Polymorphism, and Double-Primer Fluorescent Intersimple Sequence Repeat Markers. Molecular Phylogenetics and Evolution 21(3), 372-387.

Yockteng R, H.E. Ballard, G. Mansion, et al. 2003. Relationships among pansies (Viola section Melanium) investigated using ITS and ISSR markers. Plant Syst. Evol. 241: 153-170.

Zapata S. 2003. Posibilidades y potencialidad de la agroindustria en el Perú en base a la biodiversidad y los bionegocios. Comité Biocomercio Perú. Lima - Perú. 70 pp.

Zietkiewicz E., A. Rafalski \& D. Labuda. 1994. Genome fingerprinting by simple sequence repeat (SSR)-anchored polymerase chain reaction amplification. Genomics 20: 176-183. 